\title{
O Sistema de Saúde brasileiro ante a tipologia internacional: uma discussão prospectiva e inevitável
}

\author{
The Brazilian Health System in the face of international typology: a \\ prospective and inevitable discussion
}

\author{
Mauro Serapioni1,2, Charles Dalcanale Tesser ${ }^{\mathbf{2}}$
}

DOI: 10.1590/0103-11042019S504

\begin{abstract}
RESUMO Na perspectiva da análise comparada de Sistemas de Saúde (SS), este artigo analisa o SS brasileiro visando identificar estratégias promissoras para seu desenvolvimento. Metodologicamente, baseados em estudos sobre a sua formação/situação e nos seus principais componentes assistenciais e de financiamento, discutem-se suas aproximações e distanciamentos dos três tipos principais de SS: 1-baseados nos serviços nacionais universais (beveridgeanos); 2-baseados em seguros sociais obrigatórios (bismarckianos); 3-baseados em seguros privados voluntários (smithianos). O SS brasileiro é misto/segmentado, com muitos aspectos beveridgeanos, especialmente na Atenção Primária à Saúde (APS) (municipalizada e heterogênea), e smithianos (setor privado, cuidado especializado e hospitalar - insuficientes no SUS); e pouco similar aos bismarckianos. Nos seus aspectos smithianos e bismarckianos, é muito intensa a vigência da lei dos cuidados inversos, com financiamento público do setor privado para o quartil mais rico da população. Para maior racionalidade, equidade e universalidade, há que se investir nos aspectos beveridgeanos do SS brasileiro, o que não vem ocorrendo: reduzir gastos tributários em saúde, expandir e qualificar a APS via Estratégia Saúde da Família (ESF) e o cuidado especializado e hospitalar, regionalizar sua gestão, reduzindo desigualdades, e aumentar o poder de coordenação da ESF, ampliando/ modificando os Núcleos de Apoio à Saúde da Família.
\end{abstract}

PALAVRAS-CHAVE Sistemas de Saúde. Sistema Único de Saúde. Seguro saúde. Política de saúde, Brasil.

1 Universidade de Coimbra (UC), Centro de Estudos Sociais - Coimbra,

Portugal.

mauroserapioni@ces.uc.pt

2 Universidade Federal de Santa Catarina (UFSC) -

Florianopolis (SC), Brasil.
ABSTRACT In light of comparative analysis of Health Systems (HS), this article aims to discuss the Brazilian HS in order to identify promising strategies for its development. Methodologically, based on studies about its formation/situation and on its main components of assistance and of funding, the approximation and distancing from the three main types of HS are discussed: 1- those based on universal national services (Beveridgeans); 2- those based on compulsory social insurance (Bismarckian); 3- those based on voluntary private insurance (Smithians). The Brazilian HS is mixed/segmented and includes both Beveridgean aspects, especially Primary Health Care (PHC) (municipalized and heterogeneous), and Smithians elements, such as private sector, specialized and hospital care. But it is little similar to the Bismarckian HS. In its Smithian and Bismarckian aspects, the law of reverse care is more evident, with public funding from the private sector to the wealthiest quartile of the population. For greater rationality, efficiency, equity, and universality, it is necessary to invest in the Beveridgean aspects of the Brazilian HS, which does not yet occur. This means reducing health tax expenditures, expanding and qualifying both PHC, through Family Health Strategy (FHS) and specialized and hospital care, as well as regionalizing its management, reducing inequalities and increasing the coordinating role of the FHS, by expanding or modifying the Family Health Support Center.

KEYWORDS Health Systems. Unified Health System. Insurance health. Health policy, Brazil. 


\section{Introdução}

A análise comparativa no campo da saúde serve-se de tipologias de Sistemas de Saúde (SS) para identificar o contexto institucional dos cuidados de saúde e ilustrar as políticas de saúde em países diferentes ${ }^{\mathbf{1}}$. As tipologias ajudam a delinear o SS como um conjunto ideal-típico de características institucionais, com base em diversas dimensões analíticas, tais como: modalidades de financiamento dos cuidados de saúde, tipo de remuneração de médicos e outros profissionais, formas predominantes de propriedade de hospitais e serviços territoriais, modalidades de organização e prestação dos cuidados de saúde, poder das associações profissionais, papel do Estado, papel do paciente, diretrizes culturais relacionadas com a saúde e cuidados de saúde em cada país etc. Para Weber 2(106), a construção de tipos ideais tem como finalidade a compreensão da realidade, por meio da "acentuação unilateral de um ou vários pontos de vista". Nesse sentido, trata-se de uma "construção teórica pura", que não tem a pretensão de reproduzir a realidade assim como ela é, mas, ao mesmo tempo, não deve ser considerada uma construção "arbitrária e subjetiva" ${ }^{(1111)}$. A teoria weberiana dos tipos ideais, sem dúvida, tem facilitado a compreensão, a organização e a análise das diversas variedades de SS existentes em nível internacional.

Nos últimos 40 anos, os estudos comparativos dos principais modelos de SS dos países ocidentais tornaram-se um campo de pesquisa consolidado ${ }^{4-7}$. Na esteira da primeira geração de investigações comparativas sobre SS, o estudo da Organização para a Cooperação e Desenvolvimento Econômico (Organisation for Economic Co-operation and Development - OECD) ${ }^{8}$ influenciou profundamente o debate entre os estudiosos dos SS comparados. A OECD, considerando as variações no tipo de financiamento dos cuidados de saúde e as diferenças na organização das prestações, identificou três modelos básicos de SS: i) SS baseados no modelo Beveridge, com cobertura universal, financiamento proveniente dos impostos gerais e prestação pública da atenção à saúde. Esse modelo nasceu no Reino Unido, mas, por meio de um processo de difusão e adaptação, hoje é presente em muitos países europeus e extraeuropeus (Suécia, Noruega, Dinamarca, Finlândia, Islândia, Nova Zelândia, e, a partir do final dos anos 1970, Itália, Portugal, Espanha, Grécia); ii) SS baseado no modelo Bismarck, com seguros sociais obrigatórios, cobertura universal e financiado por empregadores e empregados, por intermédio de fundos de seguros sem fins lucrativos. As prestações de cuidado podem ser públicas ou privadas. Alemanha é o protótipo desse tipo de SS, mas existem vários países que têm implantado versões semelhantes (Áustria, Bélgica, França, Holanda, Suíça, Japão); iii) SS com seguros privados (modelo Adam Smith), financiado pelas contribuiç̧ões voluntárias de indivíduos e empregadores, e com prestações de cuidado de saúde predominantemente privadas. Os Estados Unidos da América(EUA) representam o protótipo desse SS, mas outros países adotaram modelos análogos que contêm elementos relevantes do sistema privado (México, Chile, Austrália etc.). Esta tipologia de SS é uma categorização descritiva bastante utilizada para avaliar a organização da assistência à saúde em diferentes países e para analisar comparativamente os processos de reforma e as políticas de saúde'.

A estes três modelos destacados pela OECD, deve-se adicionar o SS que Field6(401) definiu como sistema baseado na 'Medicina socializada', instituído na União Soviética durante a Revolução de 1917 (conhecido como modelo Shemashko), e sucessivamente difundido nos países do Leste Europeu ${ }^{9}$. Era um sistema centralizado que garantia acesso universal e financiado integralmente pelo Estado. Esse SS desenvolveu um papel importante em nível internacional até a queda do Muro de Berlim, no final da década de 1980. Cuba é um exemplo ainda presente e bem-sucedido(112) de SS baseado na medicina socializada, sem a presença do setor privado de saúde.

Para os países do Leste Europeu, a rápida transição política, econômica e social significou 
também uma transição dos SS: do modelo Shemashko a um modelo criado ad hoc por instituições internacionais (Banco Mundial e Fundo Monetário Internacional), no papel de assessores ${ }^{10}$. As mudanças envolvem todos os componentes do SS: arquitetura institucional, financiamento, organização da atenção ambulatorial, organização do cuidado hospitalar e papéis dos profissionais. Quanto ao financiamento, por exemplo, a forma recomendada e adotada na maioria dos países é o seguro social obrigatório $^{10}$. Na Rússia, coexistem, a partir de 1993, três modalidades de financiamento: a) assistência à saúde pública e universal, restrita a um programa básico (saúde materno-infantil, psiquiatria, geriatria etc.) e financiada por impostos; b) seguros sociais obrigatórios, em nível regional, gerenciados por companhias privadas; c) atenção à saúde vinculada a um seguro de saúde voluntário ${ }^{10,11}$. A grande maioria dos países com SS tipo Shemashko adotou SS baseados em seguros sociais obrigatórios (ou bismarckianos). Atualmente, o seguro social obrigatório representa a forma mais difundida de seguro saúde no mundo, mas apenas em uma minoria de países essa cobertura tem se tornado universal10.

Destarte, este artigo objetiva discutir o SS brasileiro nas suas aproximações e distanciamentos dos três tipos principais de SS acima sintetizados. Pretendemos contribuir para uma melhor clareza e orientação quanto aos desafios e direções mais (e menos) promissoras das transformações do SS brasileiro, no sentido de melhorar sua equidade, universalidade, eficiência e efetividade.

Metodologicamente, trata-se de uma análise de dados e estudos atuais e históricos sobre a formação e composição atual do SS brasileiro, nos seus principais componentes assistenciais, de provisão e de financiamento, públicos e privados. Ao invés de debater com os muitos estudos já realizados nesse campo (vide, por exemplo: Mendes ${ }^{\mathbf{1 2}}$, Ocké-Reis ${ }^{\mathbf{1 3}, \mathbf{1 4}}$, Menicucci $^{15}$, Bahia ${ }^{16,17}$, Gerschman ${ }^{\mathbf{1 8}}$, Silva ${ }^{\mathbf{1 9}}$, Gurgel Júnior et al. ${ }^{20}$, Monteiro ${ }^{21}$ ) que concordam com o caráter misto ou segmentado do
SS brasileiro, preferimos usar como referência direta de análise e estruturação do artigo os aspectos do SS brasileiro que se aproximam de cada um dos três SS ideais acima descritos, o que permite alguma simplificação e sistematização. Com isso, visamos a uma análise descritiva do SS brasileiro não exaustiva nem detalhada, mas, ao contrário, sintética e que permita vislumbrar áreas críticas e de fertilidade, desafios e potências para exploração e desenvolvimento do SS brasileiro e do Sistema Único de Saúde (SUS). Nos próximos tópicos, após uma breve explicação histórico-conceitual sobre as características gerais dos tipos ideais de SS, relacionamos os aspectos do SS brasileiro mais similares a cada um deles.

\section{O lado smithiano ou dos planos privados do SS brasileiro}

Como foi apontado, o protótipo histórico do modelo baseado nos seguros privados é representado pelo SS dos EUA. Nesse modelo, o Estado não assume a responsabilidade de garantir a proteção à saúde da população, limitando-se a proteger os grupos sociais mais vulneráveis ${ }^{9}$. Esse sistema se baseia no papel central desenvolvido pelo 'terceiro pagador', isto é, as companhias de seguros privados. $\mathrm{O}$ segurado voluntariamente paga o seguro (um plano de saúde privado) que assume o risco da doença, reembolsando o segurado pelas despesas de médicos, consultas, internações etc. Desde os anos 1950, o seguro tem sido ofertado como benefício adicional (fringe benefit) aos trabalhadores pelos empregadores ${ }^{\mathbf{2 2}}$.

O cidadão pode escolher livremente no mercado tanto o seguro como os prestadores (médicos, hospitais e laboratórios). Isso significa que a cobertura dos serviços é desigual e baseada no rendimento das pessoas. As companhias de seguro adotam comportamentos típicos das empresas que atuam no mercado: estabelecem prêmios 
de seguro de acordo com os níveis de risco do segurado, sua idade, estado de saúde etc.

A propriedade dos hospitais e clínicas é predominantemente privada ou sem fins lucrativos. No caso dos EUA, o Estado federal e os estados individuais desenvolvem uma função de controle e de regulamentação externa ao mercado de saúde, entrando no campo da prestação apenas quando se trata de estruturas pouco remuneradoras, como a psiquiatria, a atividade preventiva e a saúde pública ${ }^{22}$. $\mathrm{O}$ estatuto de profissional liberal do médico e de outros profissionais é garantido pelo forte poder das associações profissionais que controlam o acesso à profissão e o exercício dela. O papel do paciente é o de um cliente dentro de um mercado de saúde determinado pelo jogo da oferta e demanda. Do ponto de vista cultural, a orientação predominante leva a considerar a saúde como um fato individual, sendo responsabilidade do indivíduo proteger sua saúde, adquirindo a assistência à saúde como outra mercadoria ou serviço qualquer. Nesse modelo, a dominância do mercado gera ineficiência por causa da escassa regulamentação do Estado: os EUA são o país com o maior gasto em saúde no mundo e apresentam indicadores de saúde piores do que os de outros países desenvolvidos com sistemas universais?.

Nesse prisma, não se entende, realça Blank 23(415), "por que os americanos não querem mudanças significativas de um sistema obviamente quebrado?". Como aponta Martinelli24, a história da saúde nos EUA é uma história de tentativas fracassadas de introduzir programas federais de assistência à saúde: desde o intento de Theodore Roosevelt em 1912, ao de Franklin Roosevelt durante a grande crise de 1929, ao de Henry Truman em 1945, ao de Jimmy Carter em 1978, e de Bill Clinton em 1994. Somente Barack Obama, em 2010, conseguiu introduzir a reforma Affordable Care Act, definitivamente implementada em 2014. Porém, o atual presidente, Donald Trump, a está tentando desmantelar com várias estratégias judiciais e políticas, apesar dos excelentes resultados obtidos: de 2010 a 2017, o número de americanos sem seguro saúde diminuiu em 19,1 milhões, passando de 46,5 milhões para 27,4 milhões ${ }^{25}$. Isso foi o resultado da ampliação do programa Medicaid" ${ }^{1}$, instituído em 1965 pelo presidente Lyndon Johnson em favor de algumas categorias de pobres, junto com outro programa federal, o Medicare em favor de algumas categorias de idosos. O número de pessoas inscritas no Medicaid aumentou em 18 milhões, de 55 milhões, no final de 2013, para 73 milhões, em outubro de 2018, o equivalente a $22 \%$ da população norteamericana ${ }^{25}$; resultados impressionantes que devem alertar sobre um possível rebaixamento dessa reforma.

Embora os EUA representem o exemplar do SS com dominância de mercado, outros países adotaram, parcialmente ou integralmente, algumas características desse modelo. Além de México, Chile e Austrália, outros países assim como Brasil, compartilham elementos essenciais desse tipo de SS, tal como a relevância significativa de planos privados de saúde. No Brasil, desde a década de 1960, iniciaram-se os planos de saúde privados e similares, sob múltiplas modalidades (planos mutualistas de associações de categorias profissionais, seguros saúde, planos de cooperativas profissionais, planos de hospitais filantrópicos etc.). O conjunto das Unimeds, cooperativas de médicos, por exemplo, é a maior seguradora individual do mercado brasileiros dos planos de saúde. Tal mercado vem-se concentrando cada vez mais em grandes instituições privadas ${ }^{\mathbf{2 6}}$. Todavia, do ponto de vista do usuário hoje, todas as modalidades de planos ou seguro-saúde tendem a se equivaler. A cobertura de planos de saúde no Brasil, em 1998, era de 24,5\%; aumentou em 2013 para 27,9\% da população, retornando em 2019 para 24,2\%, com grande variação regional27,28.

A expansão dos planos de saúde no Brasil foi consistentemente subvencionada direta e indiretamente pela ação do Estado, sobretudo a partir da ditadura militar ${ }^{13}$. A unificação dos Institutos de Aposentadoria e Pensão (IAP) no Instituto Nacional de Previdência Social (INPS), depois Inamps, e suas sucessivas 
ampliações de cobertura se deram com investimento público diretamente no setor privado, sobretudo no setor hospitalar, para posterior compra de serviços pelo Estado. Em 1988, a criação do SUS sem o correlato investimento na rede de serviços públicos gerou, por um lado, a exclusão de trabalhadores mais bem remunerados e camadas médias de população dos serviços públicos (antes previdenciários), que procuraram o setor privado ${ }^{29}$. Por outro lado, o setor privado foi induzido, viabilizado e subvencionado pela expressiva renúncia fiscal em saúde estabelecida naquela época e em épocas mais recentes ${ }^{30}$, tanto para trabalhadores, via dedução de despesas médicas do imposto de renda, como para empregadores por meio de duas formas de incentivo: 1 - dedução dos gastos com despesas médicas com planos para os trabalhadores, consideradas despesas operacionais e abatidas do lucro líquido das empresas, base de cálculo dos impostos pagos pelas mesmas, sem teto para essas deduções; e 2 - várias outras formas de isenção fiscal para o setor privado da saúde ${ }^{\mathbf{1 3}, 30}$, por exemplo: os hospitais filantrópicos que operam seguros saúde têm deduções devido ao certificado de filantropia, as cooperativas médicas não pagam alguns tributos, há "deduções de tributos estaduais e municipais para casos específicos, decididos fora da esfera federal de governo"31(104) e há desonerações fiscais diretas para setores produtivos relacionados, como a indústria química e farmacêutica ${ }^{31}$.

No Brasil, a tradição dos planos de saúde é viabilizar o acesso dos segurados aos médicos e serviços (clínicas, hospitais etc.) credenciados de sua escolha, incluindo nessa livre escolha o acesso direto a qualquer especialista médico. Deve ser registrado que $76 \%$ do mercado de planos privados no Brasil é formado por planos coletivos, contratados coletivamente pelas empresas, em geral, e cofinanciados por patrões e empregados como um benefício atrelado ao salário e ao emprego ${ }^{32}$.

Dentro do lado smithiano do SS brasileiro está o desembolso direto do cidadão na compra de serviços e produtos de cuidado à saúde. No
Brasil, conforme a Pesquisa Nacional de Saúde de 2013, o desembolso direto ocorreu em 14,9\% dos atendimentos realizados nos últimos 15 dias, enquanto os planos de saúde cobriram 29,2\%; e o SUS, 59,9\%. Nas internações (últimos 12 meses), as proporções foram respectivamente: $10,8 \%$, $27,2 \%$ e $65,7 \% 27$. O desembolso direto correspondeu, em 2014, a 47,2 \% do gasto privado total em saúde no País33. Os gastos com desembolso direto também são subsidiados pelo Estado, por meio de deduções fiscais do Imposto de Renda de Pessoa Física (IRPF).

As deduções nos impostos acima mencionadas inserem-se dentro dos gastos tributários: gastos públicos indiretos via renúncia de receitas governamentais focalizados em setores, contribuintes ou regiões restritas, visando atender a objetivos econômicos e sociais $^{\mathbf{1 4}}$. As deduções de gastos com despesas de saúde do IRPF são o maior bloco dos gastos tributários em saúde, quase metade do total $(46,8 \%)^{34}$. Destas deduções, $70 \%$ são relativas a planos de saúde ${ }^{35}$, e o restante é referente ao desembolso direto. É importante destacar que não há teto para o volume dessas deduções, as quais beneficiam, sobretudo, os $10 \%$ mais ricos da população $0^{\mathbf{3 4}, 14}$. Somando as deduções fiscais das pessoas físicas e jurídicas com saúde e as outras renúncias fiscais no setor, chega-se a um valor dos gastos tributários em saúde correspondente a $30 \%$ do orçamento federal da saúde ${ }^{14}$. Os gastos privados em saúde com planos de saúde e desembolso direto chegam a $56,7 \%$ dos gastos totais em saúde no Brasil ${ }^{36}$. O lado smithiano do SS é o maior em gastos no Brasil, inclusive maior proporcionalmente que o dos $\operatorname{EUA}(53,4 \%)^{36}$. Ele está concentrado no quartil mais rico que tem planos de saúde, é fortemente subsidiado pelo Estado e ilustra perfeitamente a Lei dos cuidados inversos, enunciada por Hart $^{37}$, produtora de grandes iniquidades em saúde: deixado às forças do mercado, o cuidado médico tende a ser inversamente proporcional à necessidade das pessoas quem mais precisa tem menos acesso a ele, porque pode pagar menos ou não pode pagar. 
O grande subsídio estatal para a fração mais rica da população (sobretudo o decil mais rico), que já paga menos impostos, é injusta e eticamente insustentável, o que torna o lado smithiano do SS brasileiro anômalo e cruel, considerando que a ação estatal na saúde, na visão liberal e do mercado, deveria se concentrar apenas em quem não pode pagar, ou seja, na fração mais pobre da população. Adicionalmente, o fato do grosso dos trabalhadores mais qualificados e seus movimentos sindicais terem, há muito tempo, aderido aos planos privados - e de os servidores públicos e profissionais do SUS, bem como pesquisadores da saúde coletiva, usufruírem do privilégio de financiamento estatal para uso dos planos privados de saúde, além do desembolso direto - só faz acirrar o problema e a iniquidade a ele relacionados, dificultando a sua soluçãa ${ }^{38}$.

\section{O lado beveridgeano ou público do SS brasileiro}

O protótipo do sistema nacional de saúde beveridgeano é representado pelo National Health Service (NHS) do Reino Unido. O NHS foi instituído em 1948, com a aplicação do Relatório Beveridge, de 1942, que recomendava uma reorganização geral do sistema de segurança social com fim de garantir o acesso à atenção médica a todos os cidadãos, e reconhecia a saúde como um direito universal de cidadania a ser assegurado pelo Estado. Foi um ponto de virada fundamental na concepção de atenção à saúde:

um serviço de saúde integral que visava meIhorar a saúde física e mental das pessoas através de atividades de prevenção, diagnóstico e tratamento de doenças ${ }^{39(2)}$.

Tratou-se da aplicação, no campo da saúde, da filosofia do welfare state elaborada por Titmuss $^{\mathbf{4 0}}$, cujo objetivo era de aumentar o grau de cobertura de saúde da população, por meio do acesso universal e gratuito ao cuidado à saúde, com igualdade de acesso entre classes sociais e diferentes áreas territoriais, via planejamento e gestão democrática dos serviços de saúde. Financiado com base na tributação geral mediante um sistema fiscal progressivo, esse modelo de SS prevê a propriedade predominantemente pública das unidades de saúde. Os médicos (general practitioners) que atuam na Atenção Primária à Saúde (APS) no NHS são remunerados por um sistema de pagamento per capita (capitação), enquanto são considerados funcionários públicos com base em um salário todos os outros profissionais que trabalham nos hospitais e nos centros de especialidades. A maioria de hospitais e centros de saúde é de propriedade do Estado. Nesse modelo de SS, os profissionais da APS são a porta de entrada do sistema e são responsáveis pela referência para hospitais e centros de especialidades 9 . O papel do Estado é muito importante, seja para a programação e controle, seja pela prestação direta de serviços de atenção primária, secundária e terciaria. O paciente é considerado no seu "papel de cidadão-usuário de um serviço público”22(66), com direito à assistência à saúde, e o Estado tem a obrigação de garantir seu direito ao cuidado profissional em saúde-doença.

Após 30 anos de estabilidade, apesar da alternância dos partidos trabalhista e conservador no governo, com a vitória da Margaret Thatcher nas eleições gerais de 1979, uma defensora do neoliberalismo, inicia para o NHS uma fase de instabilidade e mudanças que chegam até hoje. Em 1991, Thatcher introduziu elementos de mercado e realizou uma reforma que separou as funções de compra/ aquisição de serviços das funções de prestação destes $^{39}$. O objetivo dessa reforma era criar competição entre os prestadores de serviços e melhorar a qualidade; uma competição interna ao NHS, por isso o nome de mercado interno ou quase-mercado. Quando o partido trabalhista voltou ao governo, em 1997, com Tony Blair como presidente, de todo o sistema resultante da reforma de Thatcher, ele salvou a separação entre compradores e prestadores, mas dentro de uma estrutura de programação e colaboração, e não mais na lógica de 
concorrência ${ }^{39}$. A partir de 2002, o governo Blair também interveio na estrutura do NHS, transformando hospitais em fundações que incorporaram novos atores, como representantes do governo local, universidades, mas também entidades privadas, como bancos e seguradoras. Destarte, hoje, após o governo da terceira via de Blair (1997-2007) e depois de nove anos de governo conservador (desde 2010), os processos de privatização da saúde são ainda mais acentuados no que diz respeito à provisão de serviços, enquanto o sistema de financiamento do NHS permanece público e baseado na tributação geral.

No Brasil, houve ensaios parciais de regulamentação federal da assistência médica em uma lógica seguritária e mutualista, restrita inicialmente a categorias profissionais específicas e depois a trabalhadores do mercado formal, com Carteira de Trabalho assinada. Desde a década de 1920, com as Caixas de Aposentadoria e Pensão (Caps), depois com os IAP, unificados no INPS, depois Inamps, estruturou-se uma lógica securitária que abrangia uma pequena fração da população. Tal lógica foi desmontada com a fundação do SUS, em 1988, que estabeleceu uma estruturação legal beveridgeana e declarou a universalidade do direito ao cuidado no SUS, acessado via APS ou atenção básica ${ }^{41}$, com financiamento por impostos gerais, gastos e gestão tripartites entre União (normatização), Estados (gestão da atenção hospitalar) e Municípios (administração da APS e da atenção especializada ambulatorial).

Em que pese a definição legal beveridgeana, apenas alguns aspectos do SS brasileiro são beveridgeanos: aqueles que identificamos sob a sigla SUS, que atende a aproximadamente 60 a 70\% (ou 75\%) da população, mas com gastos públicos totais de apenas $43,3 \%$ do total de gastos em saúde no País ${ }^{36}$.

O lado beveridgeano do SUS sempre foi e ainda é majoritariamente estatal na provisão de cuidados, sobretudo na APS. Esta usa a infraestrutura dos Centros de Saúde (CS) ou unidades básicas de saúde previamente existentes, até hoje insuficientes para o atendimento de toda a população. Tais serviços funcionavam anteriormente centrados na prevenção e em atividades de saúde pública, como vacinação e controle e tratamento de doenças endêmicas e agravos de relevância epidemiológica ${ }^{42}$. A APS foi organizada de forma mais efetiva tardiamente, na segunda metade da década de 1990, com o sucesso do Programa Saúde da Família, depois Estratégia Saúde da Família (ESF), que cresceu até estar presente nos últimos anos em dois terços dos CS, e mostrou melhores resultados que os $\mathrm{CS}$ sem $\mathrm{ESF}^{43}$, com mais de 42 mil equipes em $2018^{43}$, presentes em 4.995 municípios ${ }^{30}$, potencialmente responsáveis pela cobertura de $62,4 \%$ da população brasileira. Segundo a Pesquisa Nacional de Saúde de $2013,56,1 \%$ da população estava cadastrada em uma equipe de Saúde da Família ${ }^{27}$. Apesar de, atualmente, haver crescente contratação de Organizações Sociais (sem fins lucrativos) para a gestão dos CS, 95\% dos CS são de administração municipal direta" ${ }^{11}$

Há grande heterogeneidade e muitas desigualdades regionais, intermunicipais e mesmo intramunicipais em cobertura, acesso e qualidade da APS (CS e ESF), fruto da municipalização total da sua gestão. Parece não ter havido esforço ou investimento federal significativamente exitosos para reduzir tal quadro de desigualdades. Ele pode ser enfrentado por meio de reorganização regional da gestão do SUS e da APS, o que é complexo, pois implica relativizar a autonomia total dos poderes executivos municipais e estaduais na gestão da rede de serviços do SUS, bem como mudar normatizações vigentes hoje, visando reduzir disparidades intermunicipais e melhorando a qualidade e o acesso na APS e no cuidado especializado e hospitalar difusamente ${ }^{\mathbf{4 3}}$. Porém, ao contrário, a nova Política Nacional de Atenção Básica eliminou a pouca priorização anterior da $\mathrm{ESF}^{\mathbf{4 3}}$.

A provisão de cuidados especializados e hospitalares e de serviços diagnósticos sempre foi mais comprada do setor privado conveniado ao SUS. Ainda assim, o cuidado especializado no SUS, acessado via referenciamento da APS/ 
ESF, também é, na sua maioria, realizado por serviços públicos estatais, administrados pelos municípios (46,9\%) e Estados (17,9\%), sendo o restante comprado de serviços privados filantrópicos $(16,5 \%)$ ou com fins lucrativos $(14,9 \%)^{\mathfrak{1 1}}$. Sabe-se que esse é um funil no SUS, visto que há longas filas de espera para vários especialistas e certos exames diagnósticos ${ }^{31}$. Essa é a grande fatia do cuidado em que os planos de saúde privados prosperam, já que geralmente permitem acesso direto aos especialistas.

Há ainda uma fatia significativa do cuidado especializado que é de alto custo - hemodiálise, medicamentos excepcionais e para Aids, transplantes etc. -, financiada com recursos públicos federais via Fundo de Ações Estratégicas e Compensações (Faec), realizada em mais de $80 \%$ pelos serviços privados filantrópicos (26,3\%) e lucrativos (55,7\%) ${ }^{11}$.

$O$ cuidado especializado ambulatorial no SUS tem aspectos beveridgeanos subdesenvolvidos, pois herdou até hoje o formato de pagamento por procedimento do antigo Inamps (o governo federal paga por consultas e procedimentos aos municípios e estados, ou estes pagam ao setor privado). O cuidado especializado ambulatorial não recebeu empenho da gestão federal para sua estruturação, estando até hoje sem modelagem beveridgeana e sem indução normativa e financeira para sua expansão e universalização - salvo a tentativa de indução de linhas de cuidado envolvendo atenção primária, secundária e terciária para algumas condições, sem muito êxito, e a original criação dos Núcleos de Apoio à Saúde da Família (Nasf), em 2008. Nesse sentido, porém, os Nasf foram amplamente subutilizados, pois: a) foram concebidos como pertencentes à APS; b) foi priorizada sua função de apoio às equipes de Saúde da Família; c) foi despriorizada sua função de cuidado clínico (especializado), desviando-os de suas competências nucleares; d) foram-lhes atribuídas ações tipicamente das equipes de APS; e) as normativas federais excluem deles o grosso das especialidades médicas. Todavia, os Nasf poderiam ser ampliados nas suas funções, constituindo embrião de uma modelagem organizacional de cuidado especializado ambulatorial a ser induzido financeiramente pela esfera federal, fortemente coordenado pela APS, em íntima colaboração com ela e apoiando-a ${ }^{45,46}$.

O cuidado hospitalar do SUS é dividido quase igualmente na sua provisão: metade das internações ocorre em hospitais públicos de administração direta, municipais $(23,2 \%$ das internações), estaduais (22,1\%) e federais (4\%); e metade ocorre em hospitais privados conveniados filantrópicos (40,8\%) e lucrativos $(9,9 \%)^{11}$. No cuidado hospitalar, também há grande subdimensionamento da rede SUS, com o agravante de que, recentemente, há redução da rede privada conveniada ${ }^{31}$.

\section{O lado bismarckiano ou dos seguros obrigatórios do SS brasileiro}

O protótipo histórico do modelo de SS baseado nos seguros sociais obrigatórios cofinanciados é representado pelo modelo bismarckiano, criado na Alemanha, em 1883, como parte de um amplo esquema de segurança social, incluindo desemprego, maternidade, velhice etc. Trata-se do primeiro SS implementado em um país ocidental. As raízes históricas desse modelo remontam ao paternalismo reformista dos soberanos e dos principais iluministas do século XVIII, à Polícia médica (Medizinapolizei) defendida por Johann Peter Frank, que recomendou medidas estatais para a proteção da saúde individual e coletiva, e à lei prussiana de 1854 sobre seguros de saúde obrigatórios para mineiros ${ }^{24}$. Para combater a ascensão do movimento trabalhista e socialista, o governo autoritário de Bismarck atuou em duas direções: por um lado, adotou medidas repressivas proibindo a formação e a ação política das organizações socialistas; por outro, implementou um conjunto de reformas para melhorar o bem-estar dos trabalhadores de forma a garantir a paz social24. 
Esse modelo de segurança social foi preservado em suas linhas essenciais até hoje, embora tenha-se adaptado às mudanças das sociedades industriais. Inicialmente, foi desenhado para proteger os trabalhadores da indústria e as classes mais pobres (cerca de $15 \%$ da população), mas, sucessivamente foi estendido a todos os cidadãos alemães, chegando, em 2014 , a um grau de cobertura superior a $85 \%$ deles, que estão matriculados em um dos 132 seguros sociais obrigatórios ${ }^{47}$. $\mathrm{O}$ esteio desse modelo é representado pelo sistema de fundos obrigatórios de doença, instituições sem fins lucrativos financiadas pelos trabalhadores e empregadores. Até 1996, o sistema era muito corporativo, sendo que a inscrição nos fundos estava vinculada à profissão. Desde então, houve liberalização, e, portanto, há a possibilidade de escolher entre os vários seguros concorrentes entre si ${ }^{47}$. Nesses fundos, são automaticamente e obrigatoriamente segurados todos os trabalhadores e empregados da indústria, agricultura e setor terciário, pensionistas, desempregados, dependentes e familiares, e trabalhadores autônomos que não excedem um limite de rendimento estabelecido pelo Ministério da Saúde ${ }^{22}$. A contribuição paga à caixa dos fundos varia em relação à renda do empregado e corresponde a $15,5 \%$ do salário mensal (53\% do qual é pago pelo empregado, e $47 \%$, pelo empregador $)^{47}$. A obrigação de registro aplica-se a todos os empregados (e seus dependentes) com uma renda bruta mensal igual ou inferior a 4.462,60 euros. As pessoas com uma renda mensal acima desse valor podem decidir se inscrever em seguros privados, em vez dos seguros sociais obrigatórios. É o próprio Estado que paga, por meio de empréstimos específicos aos Länder (Regiões), a assistência de pessoas com deficiência, desempregados, menores e das categorias que, de outro modo, não podem ter um seguro ${ }^{47}$.

Em conformidade com o princípio da solidariedade em que se baseia o sistema de fundos obrigatórios, todas as contribuições dos assegurados são definidas com base no rendimento, e não no fator de risco individual, contrariamente aos seguros voluntários privados. Os fundos são entes de direito público, autônomos administrativa e financeiramente, e coadministrados pelos representantes dos trabalhadores e dos empregadores, que substituem o Estado na responsabilidade de garantir a saúde dos associados. Entretanto, eles permanecem sujeitos a uma estrita regulamentação e controle por parte do Estado. A propriedade das instalações de saúde é dividida entre o setor público, privado e sem fins lucrativos. Porém, a maioria dos hospitais é privada ou sem fins lucrativos, sobretudo os hospitais de reabilitação (em 2012, somente $19 \%$ eram públicos) ${ }^{47}$.

Nesse sistema, as atividades preventivas, segurança alimentar, vigilância epidemiológica e sanitária, assistência social e promoção da saúde são de responsabilidade das Regiões (Länder); e as atividades de diagnose, tratamento e reabilitação, a cargo dos fundos de seguro obrigatório ${ }^{9}$. Os cidadãos alemães têm plena liberdade para escolher o local de atendimento e o profissional, sem distinção entre clínicos gerais e médicos especialistas. Esse modelo - que não prevê o papel do gatekeeper - é típico do modelo de Bismarck, mas está mudando lentamente após uma reforma aprovada em 2004, que pretendeu fortalecer os serviços territoriais, induzir e reforçar a função filtro (gatekeeper) dos médicos de família nos seguros e reduzir a pressão sobre os hospitais. Esse modelo de SS tem uma série de variantes nacionais que refletem os diversos contextos nacionais em que foi implantado e adaptado: França, Bélgica, Áustria, Canadá, Japão e Austrália?.

No Brasil, em que pese o ensaio histórico de seguro obrigatório voltado para trabalhadores formais, com cobertura pequena, ao longo do século XX, a criação do SUS, em 1988, encerrou a trajetória inicialmente e parcialmente bismarckiana do SS brasileiro. Há, entretanto, duas similaridades entre aspectos do SS brasileiro e o SS bismarckiano. O primeiro aspecto é o acesso direto dos usuários aos especialistas nos planos de saúde voluntários e coletivos, 
que domina o imaginário social brasileiro como sendo o melhor modo de cuidado. Como a medicina brasileira acompanhou, após a década de 1950, a tendência de especialização progressiva dos EUA, e não ou muito pouco conviveu com a Medicina de Família e Comunidade (MFC) como um ramo respeitado dessa medicina, houve intensa adesão do imaginário social à medicina especializada. A grande força das especialidades médicas no mercado privado dos planos de saúde acentuou esse imaginário.

A MFC é muito pequena na sociedade brasileira, na universidade e na profissão: os médicos com residência em MFC são menos de $1 \%$ dos médicos e apenas cerca de $7,8 \%$ dos médicos da $\mathrm{ESF}^{\mathbf{4 6}, 48,49}$. Além disso, a oferta de amenidades associadas ao cuidado e a percepção de sofisticação tecnológica dura (aparelhos e exames diagnósticos) "aumentam a demanda por serviços privados em grau bem maior do que a qualidade clínica"31(93). Sabe-se que os especialistas médicos que não possuem residência em MFC manuseiam e usam muito mais tecnologia dura, contribuindo para certo senso comum de que o cuidado especializado é sistematicamente melhor. A MFC só começou a crescer no Brasil tardiamente, devido ao crescimento da APS via ESF, que gerou indução governamental de residências em MFC ao final da década de 2000. No entanto, a APS/ ESF é vista amplamente como medicina para pobres e, implicitamente, de pior qualidade ${ }^{19}$. Mais recentemente, alguns planos de saúde começaram a valorizar a MFC e a usá-la com função filtro no setor privado, o que é ainda uma minoria quase experimental, sobre o que não conhecemos dados nem sobre sua magnitude, nem sobre seus resultados.

O outro aspecto parcialmente bismarckiano do SS brasileiro é a participação estatal no financiamento dos planos de saúde de parcela da população, que poderia caracterizar o financiamento tripartido entre trabalhadores, empregadores e Estado via seguros ${ }^{50}$. Embora os planos privados brasileiros sejam voluntários, o seu financiamento parcial estatal é praticamente sistemático via gastos tributários.
Todavia, como já destacado acima, ao contrário dessa participação se dirigir às pessoas que não podem pagar (desempregados etc.), o que é regra nos SS bismarckianos, ela subsidia exclusivamente o quartil mais rico da população (declarantes do IRPF) e servidores públicos, sobretudo o decil mais rico.

Os planos e seguros de saúde brasileiros são administrados de forma privada, sem nenhuma participação dos trabalhadores na sua gestão. Também são eminentemente voluntários, embora mais de três quartos deles sejam coletivos. Além disso, a pouca e tardia regulação exercida sobre eles pelo Estado, via Agência Nacional de Saúde Suplementar (ANS), visa apenas proteger o segurado ao estabelecer regras mínimas para os contratos, sem nenhuma função ou regulação do ponto de vista de equidade, justiça e proteção social do conjunto da população. Apesar de haver grande participação estatal de subsídio e financiamento parcial a esses planos, tal participação sempre se deu no sentido de beneficiar as parcelas mais ricas da população e os empresários do setor, aumentando a iniquidade e as desigualdades em saúde do País, ao contrário do que se esperaria da atuação do Estado na seara do cuidado à saúde. Por isso, o setor de planos de saúde brasileiro tem muito pouca similaridade com os seguros obrigatórios bismarckianos, salvo talvez o acesso direto aos especialistas, hoje desestimulado e declinando pela sua tendência de maiores custos sem melhores resultados ${ }^{51}$.

\section{Considerações finais}

O Brasil tem um SS misto ou segmentado, com muitos aspectos beveridgeanos (SUS) e smithianos (setor privado), e poucas similaridades com o SS bismarckiano; com forte vigência da lei dos cuidados inversos. A parte beveridgeana é a que tem maior cobertura populacional, mas com acesso e qualidade insuficientes e desiguais na APS ${ }^{45}$. Também há insuficiência na provisão de cuidados especializados 
ambulatoriais, em que longas filas existem, assim como nas internações.

O sistema privado (smithiano) movimenta a maior parte dos gastos em saúde do País, atendendo apenas ao quartil mais rico da população. Este quartil e os servidores públicos (e do SUS) são subsidiados pelo Estado no consumo dos planos privados e no desembolso direto. O lado smithiano do SS brasileiro (com poucas/precárias semelhanças bismarckianas) padece de indefensável e iníquo financiamento público dirigido aos mais ricos, incompatível com a ética da saúde pública e do cuidado médico, que preza pela justiça social na distribuição dos recursos de cuidado.

Apesar da fundação e do crescimento do SUS beveridgeano nas últimas três décadas, sobretudo via ESF, ele foi muito limitado em financiamento e em estratégia. Apenas uma redução drástica do gasto tributário em saúde justificaria até $30 \%$ de aumento no orçamento federal na saúde. Para maior racionalidade do gasto público e universalização (na prática) do SS com equidade, há que investir no seu lado beveridgeano, sobretudo na expansão da APS (via ESF) e do seu poder de coordenação dos cuidados especializados. Retomar indutivamente a prioridade para a ESF e construir gestão regional do SUS para reduzir desigualdades são pontos férteis para avanço do SS, expandindo regionalizadamente os cuidados especializados e hospitalares, aproveitando a experiência dos Nasf, modificando-a e ampliando-a. A redução drástica ou o afastamento da necessidade de lucro é um dos fatores adicionais a favor da expansão beveridgeana do cuidado especializado e hospitalar, de maior custo. Tais focos, apenas apontados, estão esperando por inovação, desenvolvimento e investimento. Parecem ser as mais viáveis, favoráveis, sustentáveis e eticamente defensáveis direções para o aperfeiçoamento do SS brasileiro, visando equidade, universalidade e integralidade.

\section{Agradecimentos}

Mauro Serapioni agradece o apoio financeiro da Fundação Portuguesa para a Ciência e Tecnologia (FCT) CEECIND/00885/2017/ CP1402/CT0002. Charles Dalcanale Tesser agradece ao Conselho Nacional de Desenvolvimento Científico e Tecnológico (CNPq) pelo financiamento (proc. 303999/2018-7).

\section{Colaboradores}

Os autores conceberam conjuntamente o artigo. Serapioni M (0000-0002-5761-2660)* redigiu a primeira versão da introdução e da primeira parte (internacional) dos subtítulos. Tesser CD (0000-0003-0650-8289)* redigiu a primeira versão da segunda parte (nacional) dos subtítulos e as considerações finais. Serapioni M e Tesser CD realizaram igualmente a revisão crítica do conteúdo e atualização bibliográfica, sucessivas revisões da redação e aprovação da versão final do manuscrito. 


\section{Referências}

1. Burau V, Blank RH. Comparing Health Policy: An Assessment of Typologies of Health Systems. J Comp Pol Anal. 2006; 8(1):63-76.

2. Weber M. Sobre a teoria das ciências sociais. São Paulo: Moraes; 1991.

3. Sell CE. Sociologia clássica. Marx, Durkheim e Weber. Petrópolis: Vozes; 2010.

4. Roemer M. National Health Systems of the World, New York: Oxford University Press; 1991.

5. Moran M. Understanding the welfare state: the case of health care. Br J Polit Int Relat, 2000; 2:135-160.

6. Field MG. The health system and the polity: A contemporary American dialectic. Soc Sci Med Med Psychol Med Sociol. 1980; 14(5):397-413.

7. Wendt C, Frisina L, Rothgang H. System Types: A Conceptual Framework for Comparison. J Soc Policy. 2009; 43(1):70-90.

8. Organização para a Cooperação e Desenvolvimento Económico. Financing and Delivering Health Care: A comparative analysis of OECD Countries. Paris: OECD; 1987.

9. Lobato LVC, Giovanella L. Sistemas de Saúde: origens, componentes e dinâmica. In: Giovanella L, Escorel S, Lobato LVC, et al. Políticas e Sistema de Saúde no Brasil. 2. ed. Rio de Janeiro: Fiocruz; 2012.

10. Macciocco G. Politica, salute e sistemi sanitari. Le riforme dei sistemi sanitari nell'era della globalizzazione. Roma: Il Pensiero Scientifico; 2009.

11. Marques RM, Piola SF, Roa AC, organizadores. Sistema de saúde no Brasil: organização e financiamento. Rio de Janeiro: ABrES; Brasília, DF: Ministério da Saúde; OPAS/OMS no Brasil; 2016.

12. Mendes EV. As políticas de saúde no Brasil nos anos
80: a conformação da reforma sanitária e a construção da hegemonia do projeto neoliberal. In: Mendes EV, organizador. Distrito sanitário: o processo social de mudança das práticas sanitárias do sistema único de saúde. São Paulo: Hucitec-Abrasco; 1993. p. 19-91.

13. Ocké-Reis CO. O Estado e os planos de saúde no Brasil. Rev Serviço Publico. 2000; 51(1):123-147.

14. Ocké-Reis CO. Sustentabilidade do SUS e renúncia de arrecadação fiscal em saúde. Ciênc. Saúde Colet. 2018; 23(6):2035-2042.

15. Menicucci TMG. Relação público-privado no sistema de saúde brasileiro: opacidade cognitiva e analítica. Ciênc. Saúde Colet. 2008; 13(5):1398-1408.

16. Bahia L. As contradições entre o SUS universal e as transferências de recursos públicos para os planos e seguros privados de saúde. Ciênc. Saúde Colet. 2008; 13(5):1385-1397

17. Bahia L. O sistema de saúde brasileiro entre normas e fatos: universalização mitigada e estratificação subsidiada. Ciênc. Saúde Colet. 2009; 14(3):753-762.

18. Gerschman S. Políticas comparadas de saúde suplementar no contexto. Ciênc. Saúde Colet. 2008; 13(5):1441-1451.

19. Silva SF. Interfaces entre a gestão do SUS e a regulação de planos privados de saúde. Ciênc. Saúde Colet. 2008; 13(5):1403-1406.

20. Gurgel Júnior GD, Sousa MC, Oliveira SRA, et al. The National Health Services of Brazil and Northern Europe: universality, equity, and integrality - time has come for the latter. Int J Health Serv. 2017; 47(4):690-702.

21. Monteiro MG. As mudanças de direção nas políticas públicas: atores e estratégias ocultas na implementação do Sistema Único de Saúde. In: Fleury S, organizadores. Teoria da reforma sanitária: diálogos críticos, Rio de Janeiro: Fiocruz; 2018. 
22. Giarelli G. Malessere della medicina. Un confronto internazionale. Milão: Franco Angeli; 2003.

23. Blank RH, Transformation of the US Healthcare System: Why is change so difficult? Current Sociology. 2012; 60(4):415-426.

24. Martinelli A. Salute e sistemi sanitari occidentali. In: Donati PP, organizador. La sociologia sanitaria. Dalla sociologia della medicina alla sociologia della salute. Milão: Franco Angeli; 1983.

25. Macciocco S. Sono pazzi quiesti americani. Salute Internaziponale.info [internet]. 2019. [acesso em 2019 abr 25]. Disponível em: http://www.saluteinternazionale.info/2019/01/sono-pazzi-questi-americani/.

26. Araújo AAS, Silva JRS. Análise de tendência da sinistralidade e impacto na diminuição do número de operadoras de saúde suplementar no Brasil. Ciênc. Saúde Colet. [internet], 2018 [acesso em 2019 out 29]; 23(8):2763-2770. Disponível em: http://dx.doi. org/10.1590/1413-81232018238.20572016.

27. Viacava F, Bellido JG. Condições de saúde, acesso a serviços e fontes de pagamento, segundo inquéritos domiciliares. Ciênc. Saúde Colet. 2016; 21(2):351-370.

28. Agência Nacional de Saúde Suplementar (ANS) [internet]. [acesso em 2019 nov 6]. Disponível em: http:// www.ans.gov.br/perfil-do-setor/dados-gerais.

29. Faveret Filho P, Oliveira PJ. A universalização excludente: reflexões sobre as tendências do sistema de saúde. Planejamento e Políticas Públicas. 1990; 3(1):139-162.

30. Bahia L, Scheffer M. O SUS e o setor privado assistencial: interpretações e fatos. Saúde debate. 2018; 42(esp3):158-171.

31. Santos IS, Santos MAB, Borges DCL. Mix público-privado no sistema de saúde brasileiro: realidade e futuro do SUS. A saúde no Brasil em 2030 - prospecção estratégica do sistema de saúde brasileiro: estrutura do financiamento e do gasto setorial. Rio de Janeiro: Fiocruz/Ipea/Ministério da Saúde/Secretaria de Assuntos Estratégicos da Presidência da República; 2013. (v. 4).

32. Santos RT. SUS: um novo capítulo de lutas. Ciênc. Saúde Colet. 2018; 23(6):1719-1720.

33. Figueiredo JO, Prado NMBL, Medina MG, et al. Gastos público e privado com saúde no Brasil e países selecionados. Saúde debate. 2018; 42(esp2):37-47.

34. Dain S, Ocké-Reis CO, Geiger F, et al. Regime Fiscal dos Estabelecimentos e Empresas de Planos e Seguros Privados de Saúde: legislação fiscal, mensuração dos gastos tributários, relações com a política fiscal, impactos sobre a equidade e relações com a regulação. Relatório Final [internet]. Agência Nacional de Saúde Suplementar. 2015. [acesso em 2018 dez 18]. Disponível em: https://saudeamanha.fiocruz.br/wp-content/uploads/2016/12/relatorio-final-0-Regime-Fiscal-Planos-Privados.pdf.

35. Receita Federal. Grandes Números IRPF - Detalhamento das Deduções com Despesas Médicas [internet]. [acesso em 2019 fev 1]. Disponível em: http://receita.economia.gov.br/dados/receitadata/estudos-e-tributarios-e-aduaneiros/estudos-e-estatisticas/11-08-2014-grandes-numeros-dirpf/relatorio-despesas-medicas-dos-ac-2007-a-2016.pdf/ view.

36. Organização para a Cooperação e Desenvolvimento Económico. Health Statistics 2018 [internet]. [acesso em 2019 fev 11] Frequently requested data. Disponível em: https://www.oecd.org/els/health-systems/ OECD-Health-Statistics-2018-FrequentlyRequested-Data.xls.

37. Hart JT. The inverse care law. The Lancet. 1971; 27:405-412.

38 Tesser CD, Serapioni M. O longo caminho para a universalização do Sistema Único de Saúde. Ciênc. Saúde Colet [internet]. 2019 [acesso em 2019 out 21]; 23(6). Disponível em: http://cienciaesaudecoletiva.com.br/ artigos/obstaculos-a-universalizacao-do-sus-gastos-tributarios-demandas-sindicais-e-subsidio-estatal-de-planos-privados/17334. 
39. Macciocco G. I 70 anni del NHS. Salute Internaziponale.info, 2018 [internet]. [acesso em 2019 abr 30]. Disponível em: http://www.saluteinternazionale. info/2018/05/i-70-anni-del-nhs/.

40. Titmuss RM. Essays on the Welfare State. Surrey: Unwin Brothers; 1963.

41. Medina MG. Dialogando com os autores: concordâncias e controvérsias sobre atenção primária à saúde no Brasil. Cad. Saúde Pública [internet]. 2018 [acesso em 2019 out 20]; 34(8):e00116118. Disponível em: https://doi.org/10.1590/0102-311X00116118.

42. Campos CEA. As origens da rede de serviços de atenção básica no Brasil: o Sistema Distrital de Administração Sanitária. Hist Ciênc Saúde. 2007; 14(3):877906.

43 Tesser CD, Norman AH, Vidal TB. Acesso ao cuidado na atenção primária à saúde brasileira: situação, problemas e estratégias de superação. Saúde debate. 2018; 42(espl):361-378.

44. Santos L, Campos GWS. SUS Brasil: a região de saúde como caminho. Saúde e Soc. 2015; 24(2):438-46.

45. Tesser CD. Núcleos de Apoio à Saúde da Família, seus potenciais e entraves: uma interpretação a partir da atenção primária à saúde. Interface (Botucatu) [internet]. 2017; 21(62):565-578. Disponível em: http:// dx.doi.org/10.1590/1807-57622015.0939.

46. Tesser CD, Poli Neto P. Atenção especializada ambulatorial no Sistema Único de Saúde: para superar um vazio. Ciênc. Saúde Colet. 2017; 22(3):941-951.
47. Bellini I. Il sistema sanitario tedesco [internet]. 2014. [acesso em 2019 abr 25]. Disponível em: http://www. saluteinternazionale.info/2014/09/il-sistema-sanitario-tedesco/.

48. Scheffer M, coordenador. Demografia Médica no Brasil 2018 [internet]. São Paulo: Departamento de Medicina Preventiva da Faculdade de Medicina da USP; Conselho Regional de Medicina do Estado de São Paulo; Conselho Federal de Medicina; 2018. [acesso em 2019 abr 30]. Disponível em: http://www.flip3d. com.br/web/pub/cfm/index10/?numero=15\&edicao $=4278 \#$ page $/ 8$.

49. Augusto DK, David L, Oliveira DOPS, et al. Quantos médicos de família e comunidade temos no Brasil? Rev Bras Med Fam Comunidade [internet]. 2018 [acesso em 2019 out 29]; 13(40):1-4. Disponível em: http://dx.doi.org/10.5712/rbmfc13(40)1695.

50. Dia Filho PPS. Neocorporativismo e Saúde Suplementar: as bases sociais da privatização da Saúde no Brasil. 2016. [tese]. Rio de Janeiro: Escola Nacional de Saúde Pública Sergio Arouca, Fundação Oswaldo Cruz; 2016. p. 305.

51. Homa L, Rose J, Hovmand PS, et al. A participatory model of the paradox of Primary Care. Ann Fam Med. 2015; 13(5):456-65.

Recebido em 08/05/2019

Aprovado em 20/08/2019

Conflito de interesses: inexistente

Suporte financeiro: Fundação Portuguesa para a Ciência e

Tecnologia (FCT) - CEECIND/00885/2017/CP1402/CT0002 e

Conselho Nacional de Desenvolvimento Científico e Tecnológico

(CNPq) - (proc. 303999/2018-7) 